\title{
Utilizing the Classical Overhung Beam to Provide a Range of Design, Analysis, and Test Experiences to an Experimental Mechanics Class
}

\author{
William K. Szaroletta \\ Purdue University, West Lafayette, Indiana
}

\begin{abstract}
:
The classical overhung beam is widely utilized in product design, machine design, and architectural design. An element of an upper level experimental mechanics class utilizes the overhung beam to provide a rich source for design, analysis, and test experiences.

Although overhung beam theory has been taught at both the lower and upper levels of Purdue University's Mechanical Engineering Technology curriculum, there has been little opportunity for hands-on experimental and finite element analysis verification of overhung beam theory. To fill that void, several experimental mechanics class projects were devised and successfully implemented by the author and these experiences are documented in this paper.
\end{abstract}

\section{Introduction:}

This paper describes a series of reproducible projects, including discussion of the major benefits of utilizing the overhung beam: flexibly supporting multiple experiments with similar set-ups; providing a relatively simple and effective means towards achieving a repeatable, uniform distributed loading in a laboratory environment (through gravity and beam material density); and providing a relatively low cost platform to support this suite of experiments.

The uniform distributed loading projects described in this paper include a simply-supported beam with the beam supports at the beam extremities, an overhung beam with eight different offset distances provided by beam support placement, and an investigation to analytically and experimentally find the optimal offset distance. These projects provided mechanics students with practical experience with theory, optimal design, solid modeling, finite element analysis (FEA), experiment design, and hands-on testing experience. The results of these experiments, which the students presented to the entire class, demonstrate good correlation among the theory, FEA, and experiment.

\section{Simply Supported and Overhung Beam Theory and Analysis:}

Analysis of the classical overhung beam is begun with routine analysis of the simply supported beam with a distributed load, w, along the entire length. Mechanics theory is reviewed and Experimental Mechanics students are expected to construct a Loading, Shear, and Moment diagrams as shown in below in Figure 1. These same students are introduced to the subject by being able to visualize deflection through the utilization of an 0.5 " thick by 2 " wide by 96 " long Aluminum test beam. Instructor demonstration that a simply supported beam can be converted to an overhung beam by moving the supports towards the center of the beam in unison shows graphically how the overhung part reduces the center deflection; an event that clues the students to the fact that the stress could possibly be less at this midpoint location as well.

From a design of experiment point of view, application of loads can require extra weights or equipment. Providing a uniform distributed load with external weights or equipment can be 
done, but there are extra parts required. With the simply supported and overhung beam in this experiment, gravity works with the material to produce the uniform distributed load. This fact reduces the complexity (and cost) of the experiment required to perform the experiment.

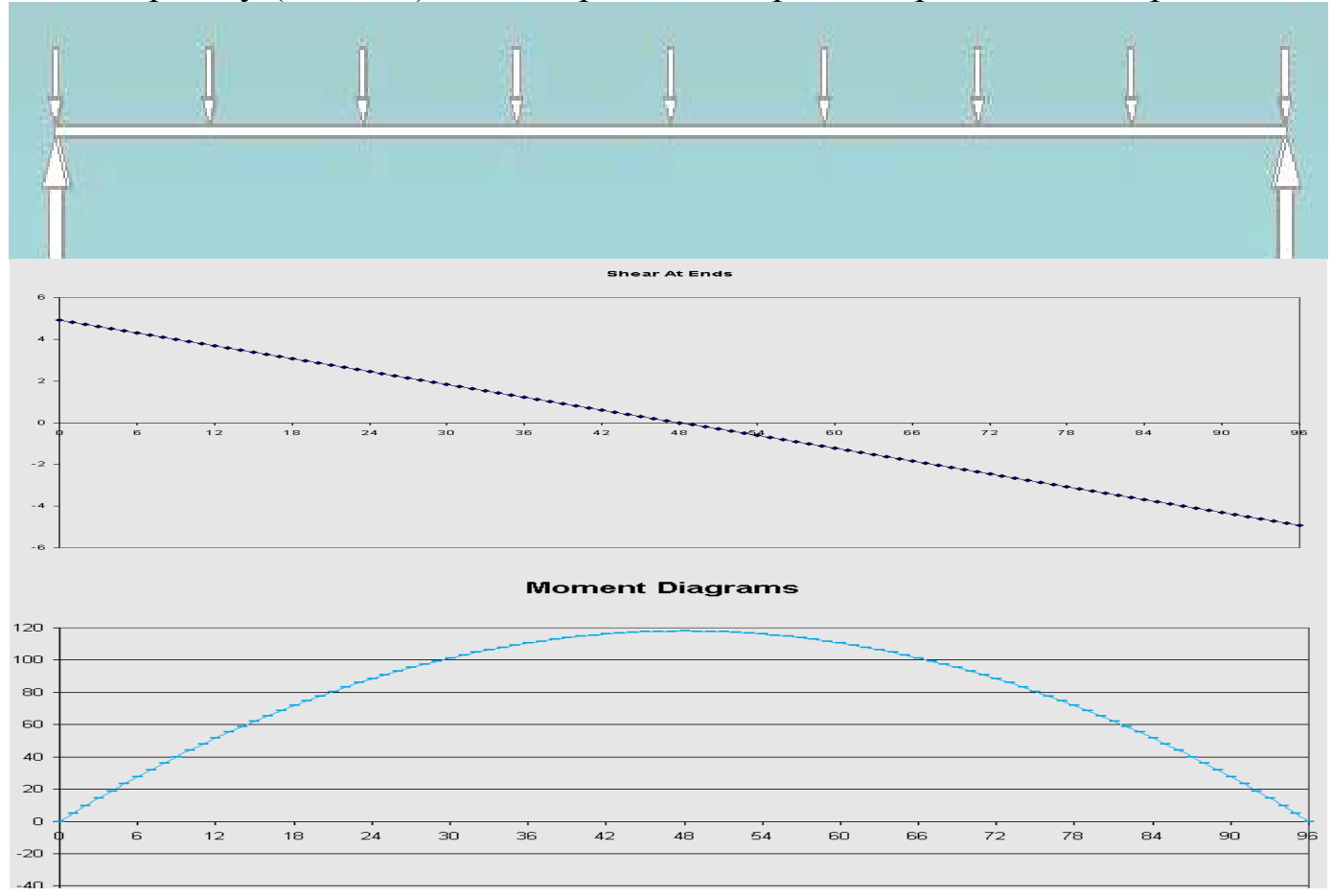

Figure 1: Free Body, Shear, and Moment diagrams for simply supported beam with distributed load

Both traditional and Finite Element Analysis (FEA) were performed on the beams and the results compared with experimental results with good (but could be better) correlation of roughly $90 \%$. The FEA on both the simply supported and overhung beam is shown below in Figure 2, with one of the supports modeled as a sliding constraint and the other a fixed constraint. Figure 3 depicts a solid model of the overhung beam with test pads at strain gage locations.
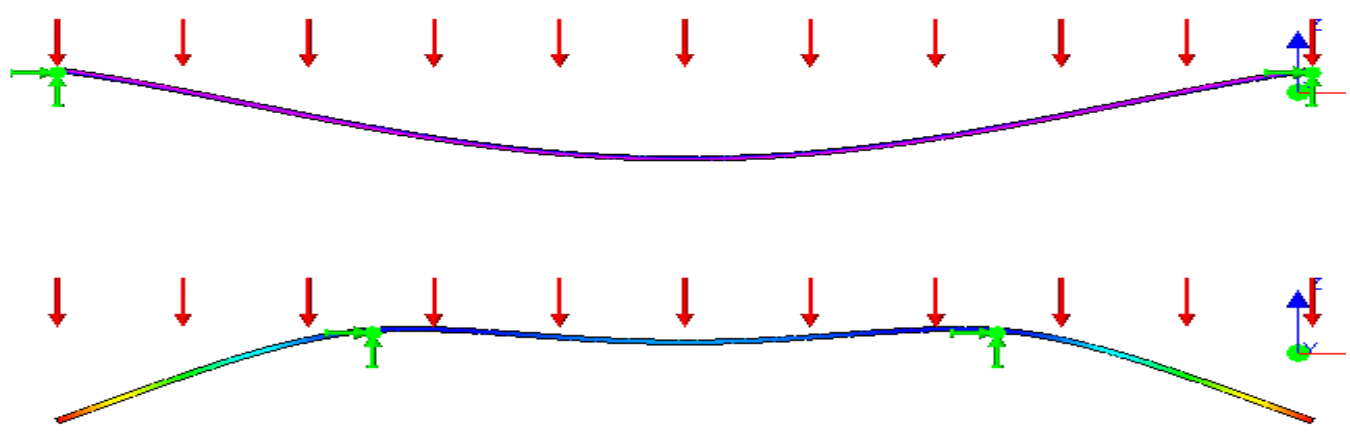

Figure 2: Finite Element Analysis (FEA) of simply supported beam with distributed load and overhung beam performed by students using COSMOS DesignSTAR ${ }^{\text {TM. }}$ 


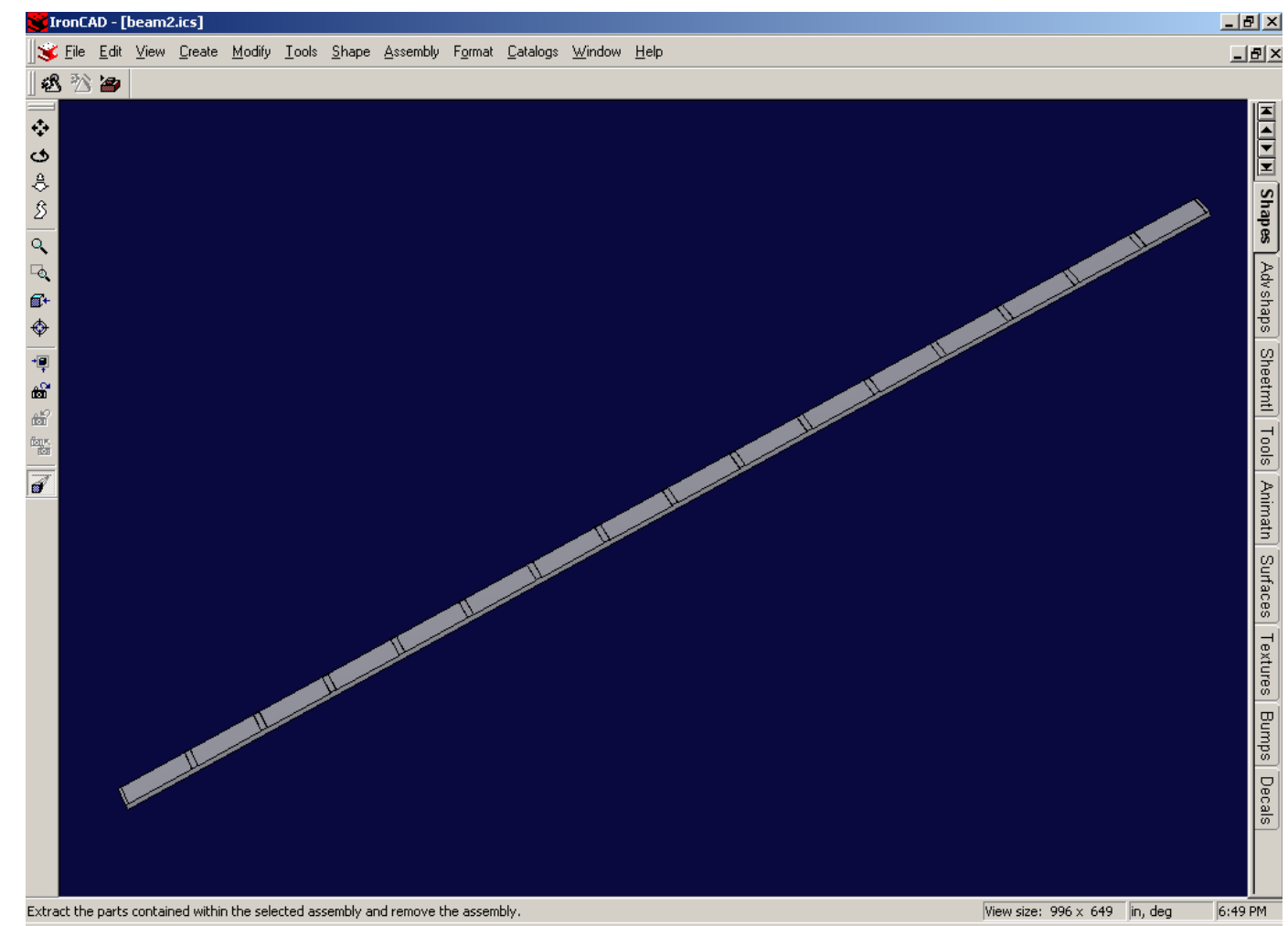

Figure 3: Solid model of overhung beam with strain gage mounting points shown. Students utilized IronCAD ${ }^{\mathrm{TM}}$ for this modeling work.

\section{Spreadsheet Solver Software and Analysis Utilized to Determine Optimal Offset Distance:}

The spreadsheet solver method is becoming widely known and popular among Purdue University undergraduate students. For this paper, the Microsoft Excel ${ }^{\mathrm{TM}}$ add-in package Solver ${ }^{\mathrm{TM}}$ is utilized, although other packages are readily available, including MATLAB ${ }^{\mathrm{TM}}$, MathCAD $^{\mathrm{TM}}$, Mathematica ${ }^{\mathrm{TM}}$, Maple ${ }^{\mathrm{TM}}$, etc. The intent for the students was to analyze how the offset distance affects the stress at the supports and the midspan. Clearly, in the case of the simply supported configuration, the flexural stress is maximum at midspan and minimum at the supports. Students learn through doing and analysis that the flexural stress at midspan decrease (and the support flexural stress increases) as the supports are moved towards midspan. The optimal offset distance is defined by Shigley to be where these two flexural stresses are equal in value, though opposite in sense. Solver ${ }^{\mathrm{TM}}$ is utilized to accomplish this as shown in Figure 4. The target cell which is the difference of the midspan and support moments is driven towards zero by varying the offset distance in this configuration. In parallel, the students did an algebraic solution to the optimal offset distance of the overhung beam, which is shown below in Figure 5.

In Purdue University prerequisite courses MET 111, Statics, and MET 211, Applied Strength of Materials, students have previously had extensive experience with shear, moment, slope, and deflection diagram mathematics. The overhung beam provided them with an interesting twist on this previous work by requiring them to solve for the slope diagram's constants of integration between tip and support and support and midspan using the fact that the slope is constant at the support. The mathematical development is shown in Figure 6 below. 


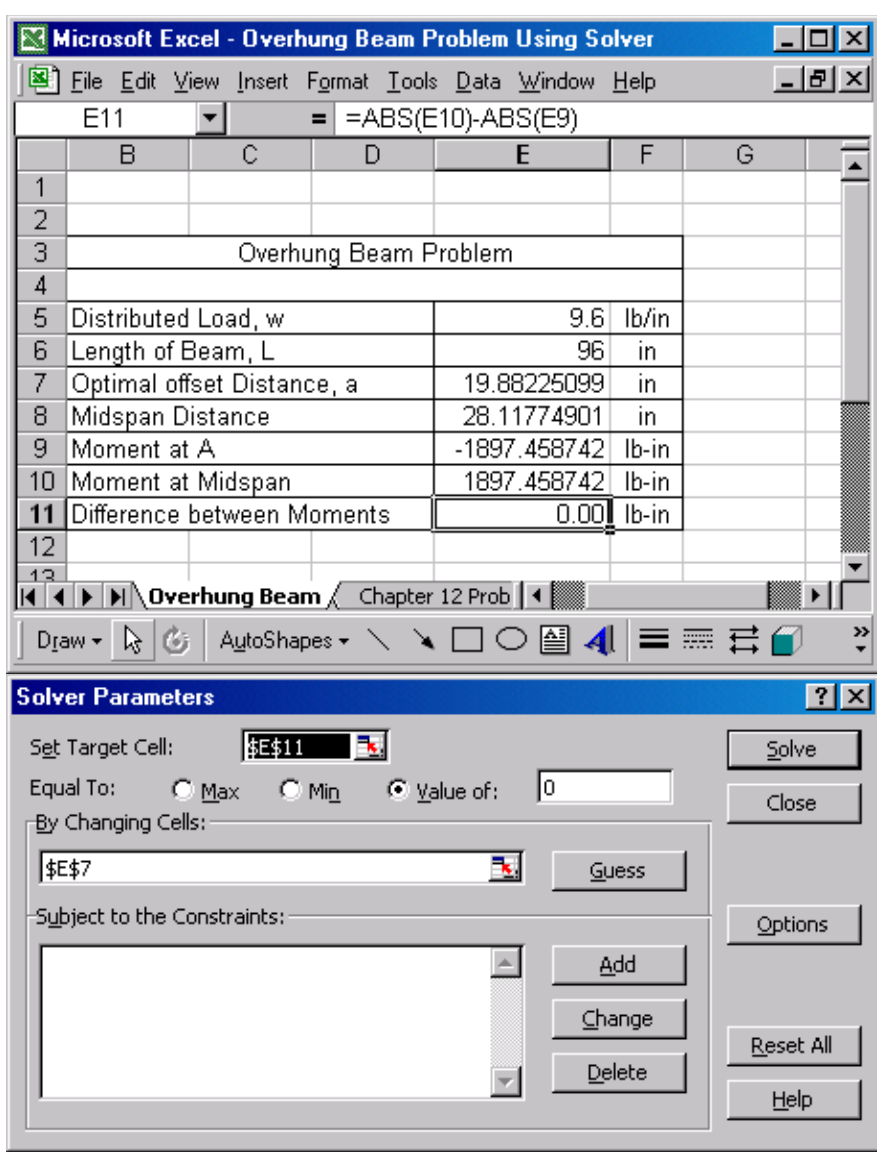

Figure 4: Example of students utilizing Solver ${ }^{\mathrm{TM}}$ to find the optimal offset distance of the overhung beam. For the example beam the distance is 19.9" which agrees exactly with theory and closely ( $9 \%$ error) with experiment.

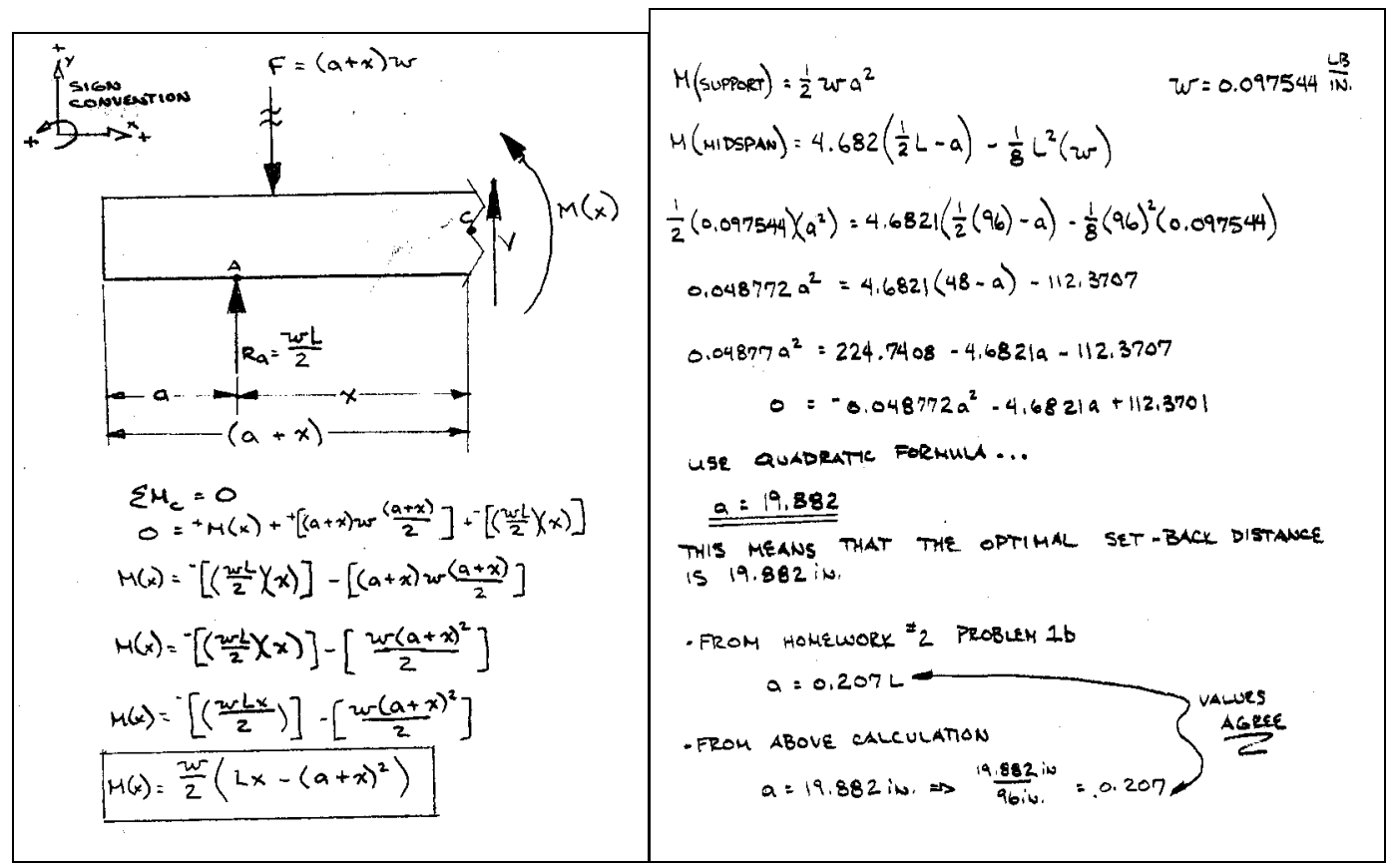

Figure 5: Example of student work to determine optimal offset distance of overhung beam. 


$$
\begin{aligned}
& V_{A B(P)}=-\omega \rho \\
& m_{A B(p)}=\frac{-w \rho^{2}}{2} \\
& E I \sigma_{A B(l)}=\frac{-\omega f^{3}}{6}+\left[\frac{-\omega L^{3}}{24}+\frac{\omega L^{2} a}{4}-\frac{\omega L a^{2}}{4}\right] \\
& E I_{y_{A B}(P)}=\frac{-\omega \rho^{4}}{24}+\left[\frac{-\omega L^{3}}{24}+\frac{\omega L^{2} a}{4}-\frac{\omega L a^{2}}{4}\right] \rho+\left[\frac{\omega a^{4}}{24}+\frac{\omega L a^{3}}{4}-\frac{\omega L^{2} a^{2}}{4}+\frac{\omega L^{3} a}{24}\right] \\
& \text { where } a=\text { offset distance (in) } \\
& l=\text { Query point from end of beam (in) } \\
& \omega=\text { distributed Load ( } 16 / n) \\
& L=\text { Beam Length (in) } \\
& V_{S S(x)}=V_{B C(x)}=-w(x)+\frac{w L}{2} \\
& M_{s s}(x)=M_{\theta C}(x)=-\frac{\omega x^{2}}{2}+\frac{\omega L x}{2} \\
& E I \theta_{S S(x)}=E I \theta_{B C(x)}=\frac{\omega}{2}\left[\frac{-x^{3}}{3}+\frac{L x^{2}}{2}-a x^{2}-a^{2} x+\left(\frac{-L^{3}}{12}+\frac{L^{2} a}{2}-\frac{L a^{2}}{2}-\frac{a^{3}}{3}\right)\right] \\
& E I_{y_{s S}(x)}=E I_{y_{B C}(x)}=\frac{\omega}{2}\left[\frac{-x^{4}}{12}+\frac{L x^{3}}{6}-\frac{a x^{3}}{3}-\frac{a^{2} x^{2}}{2}+\left(\frac{-L^{3}}{12}+\frac{L^{2} a}{2}-\frac{L a^{2}}{2}-\frac{a^{3}}{3}\right) x\right] \\
& \text { where } \omega=\text { distributed Load ( } \mathrm{b} / \mathrm{m}) \text { For } B C \text { (overhung) } \\
& \begin{array}{l}
\omega=\text { Beam Length (in) } \quad x=\text { Query Distance from (in) } \\
\text { For SS }
\end{array} \\
& x=\text { Query Distuce from (in) } \\
& a=O \text { (in) }
\end{aligned}
$$

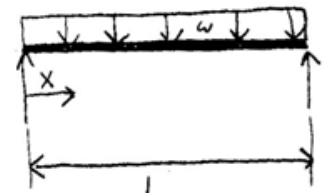

$$
\begin{aligned}
& a=\text { offect distance (in) }
\end{aligned}
$$

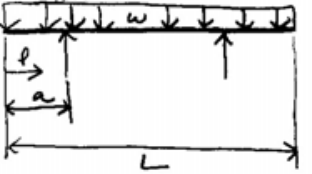




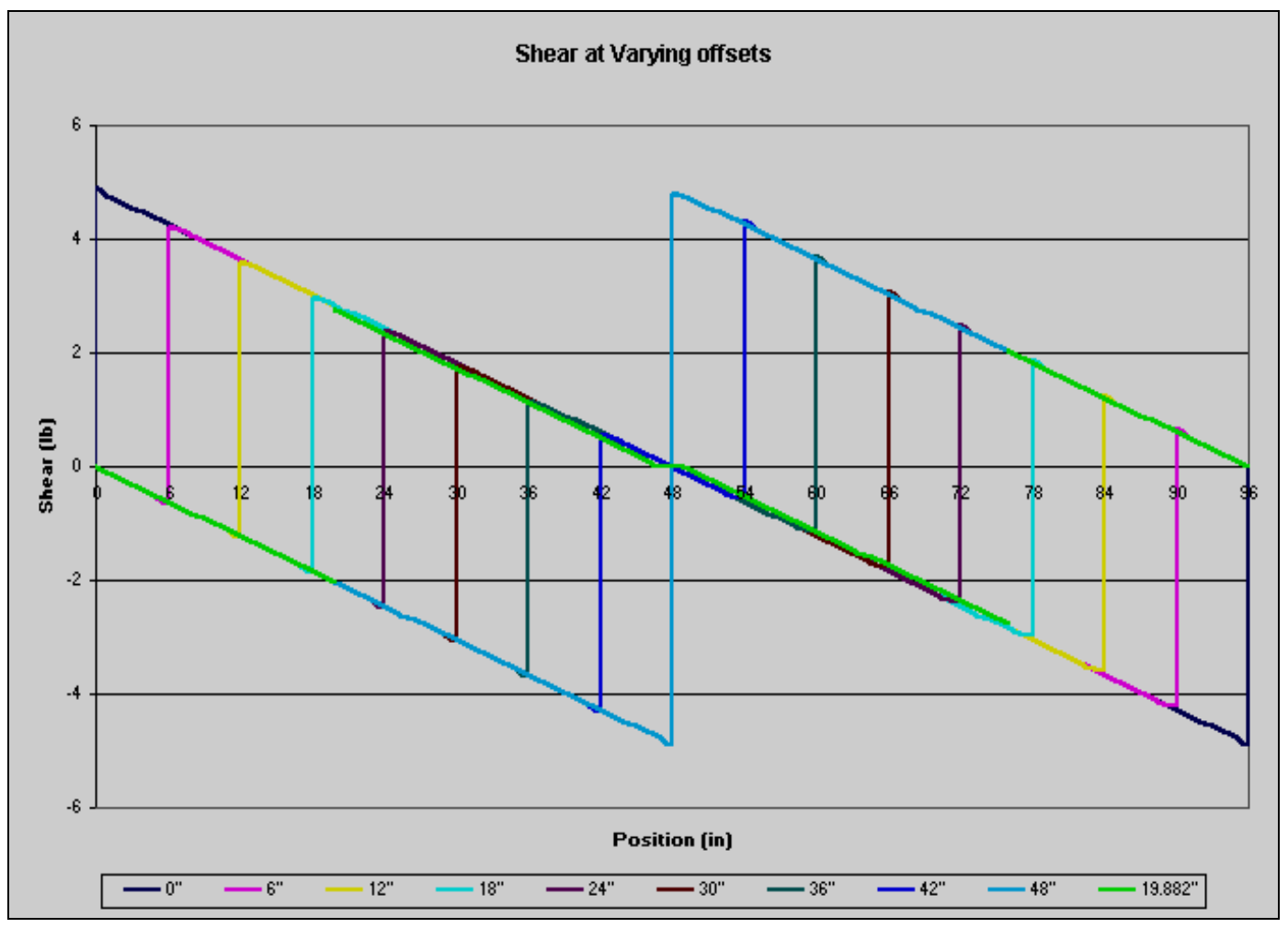

Figure 7: Theoretical Shear Diagrams for the Overhung Beam with various offsets

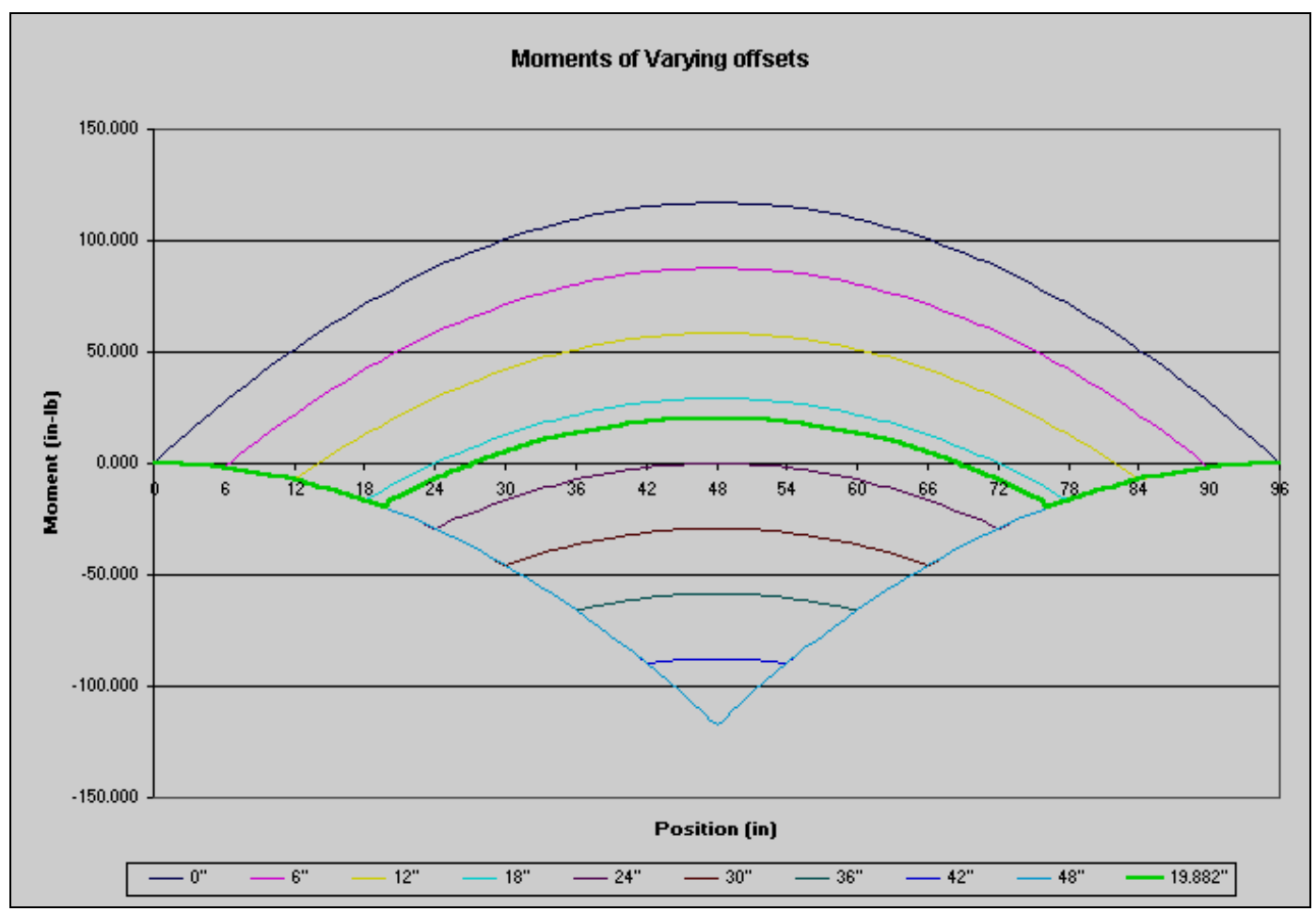

Figure 8: Theoretical Moment Diagrams for the Overhung Beam with various offsets 


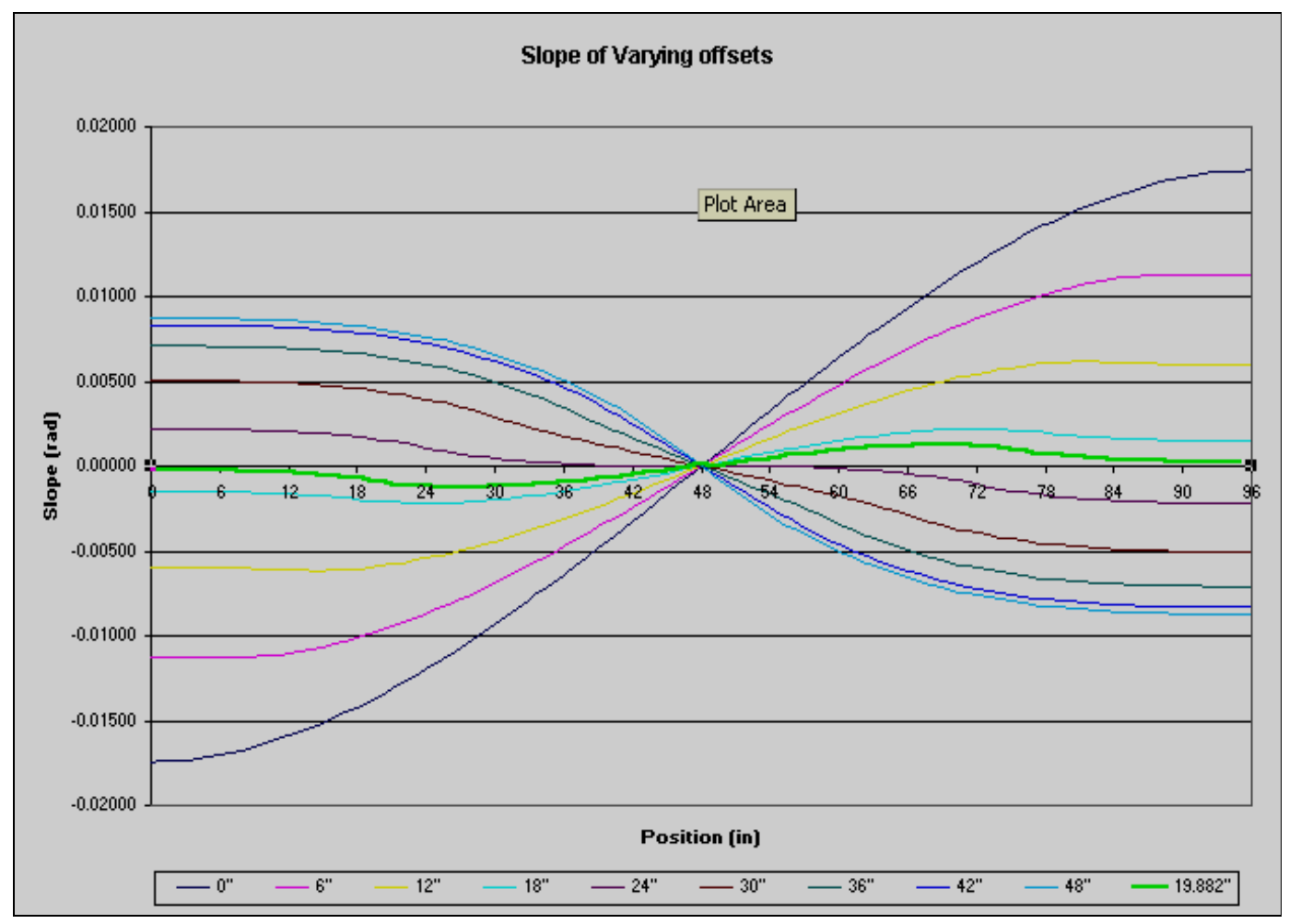

Figure 9: Theoretical Slope Diagrams for the Overhung Beam with various offsets

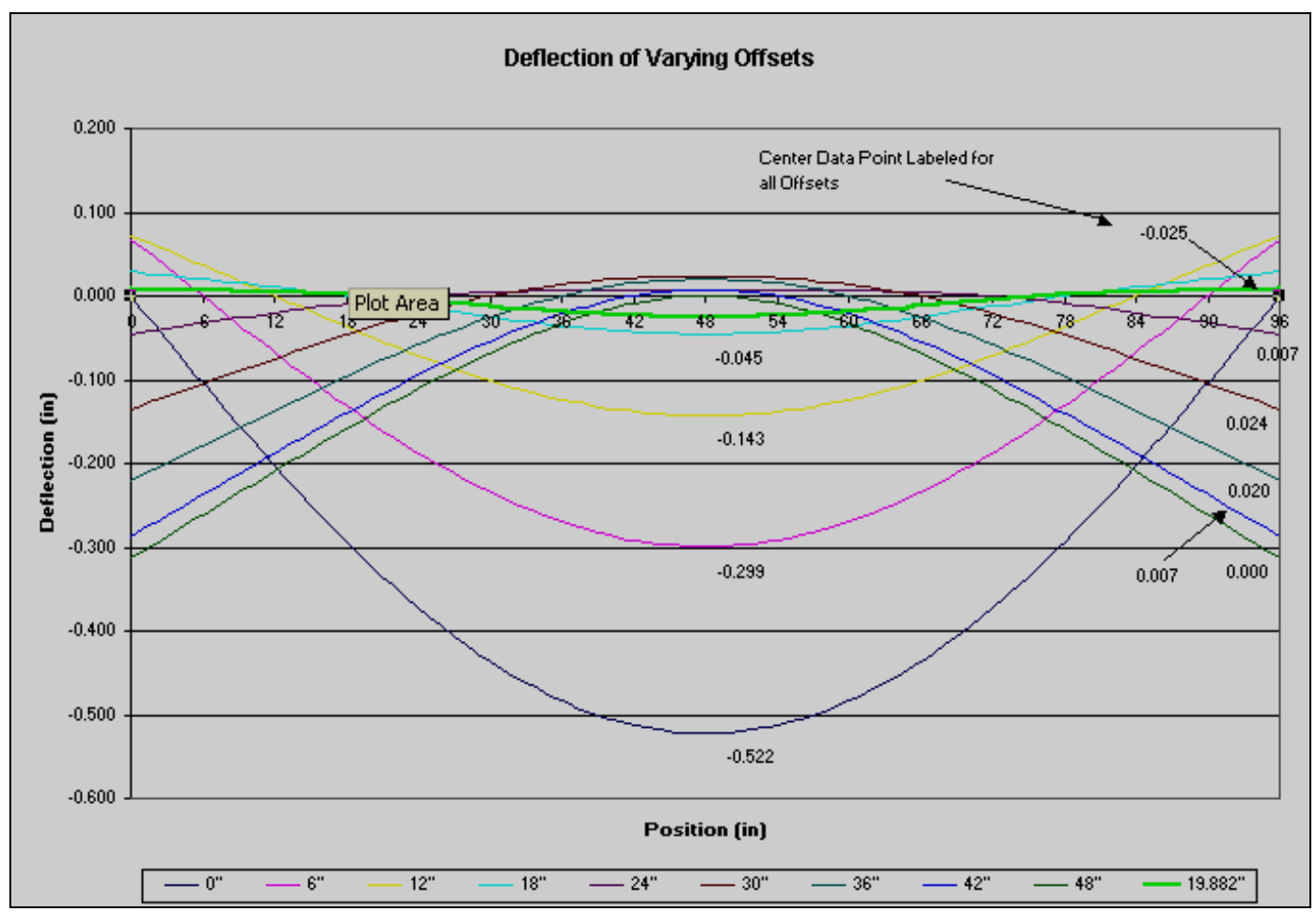

Figure 10: Theoretical Deflection Diagrams for the Overhung Beam with various offsets

Through evaluation of these diagrams, multiple opportunities for student learning present themselves including: how and why the strain and stress diagrams would match the moment diagram's shape, how and why the tip deflection is sometimes negative, sometimes positive, what characteristics of the diagrams for the optimally offset beam highlight this as a special case. 


\section{Experimental Overview:}

Student teams mounted strain gages on beam specimens at equally-spaced locations of 6", 12", $\ldots, 48^{\prime \prime}$ and these strain gages were connected through a Vishay SB-10 ${ }^{\mathrm{TM}}$ to a Vishay P-3500 ${ }^{\mathrm{TM}}$ as shown in the wiring diagram and picture in Figure 11 below.

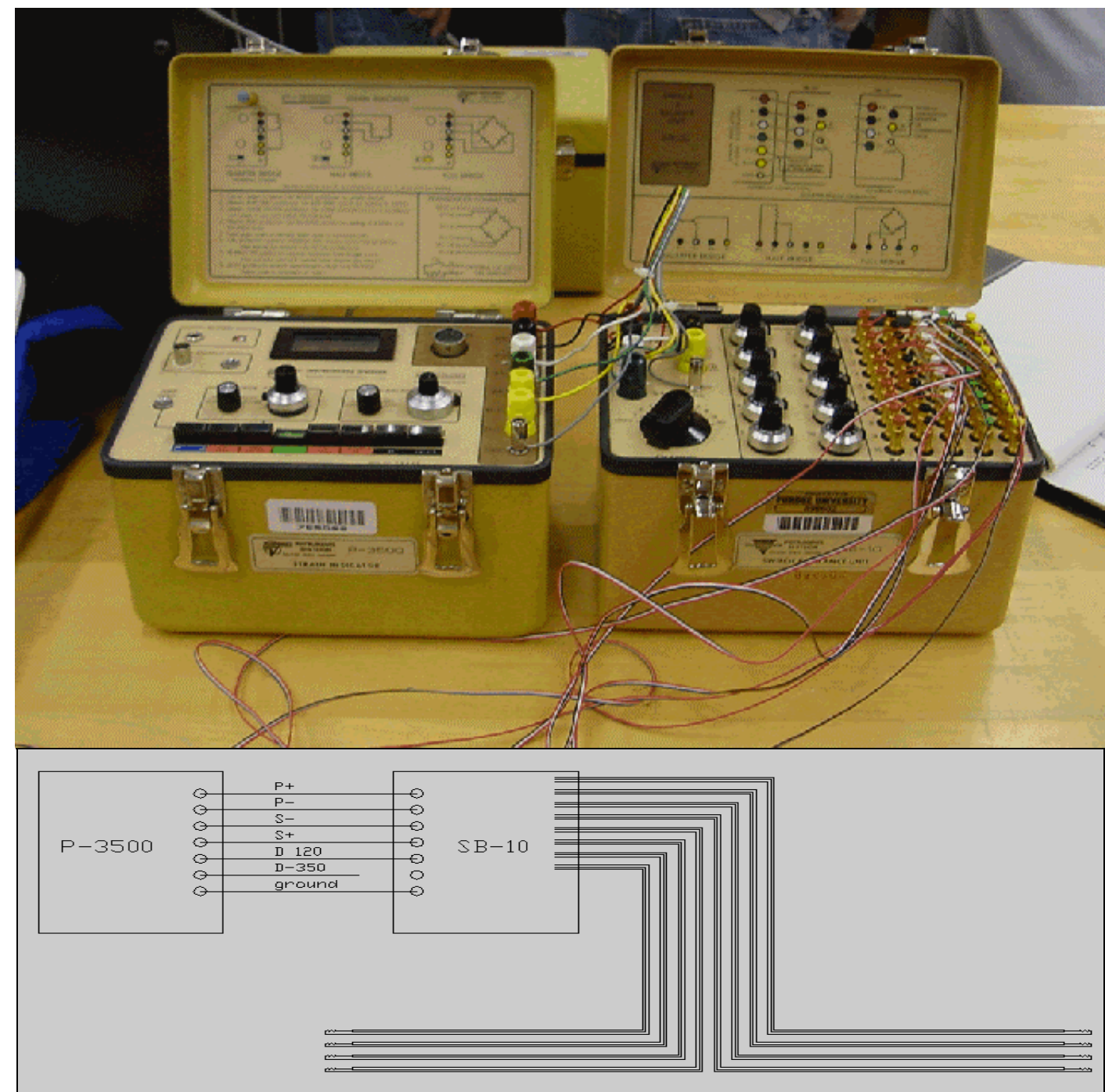

Figure 11: Vishay $P 3500$ on left and SB-10 on right. This arrangement allowed eight strain gages to be tested for each configuration. Connection diagram is shown on bottom.

An exciting part of this project from an ABET perspective was the opportunity for including an open-ended design component. To account for beam warpage in the strain and deflection measurement phase, the instructor required the teams to measure strain and deflection with the strain gages facing up and subsequently facing down. Clearly the strain gage would be crushed by the support with this method in the strain-gage-down test configuration. The teams all designed similar fixtures with a relief to protect the strain gages. The before and after designs are shown below in Figure 12. 


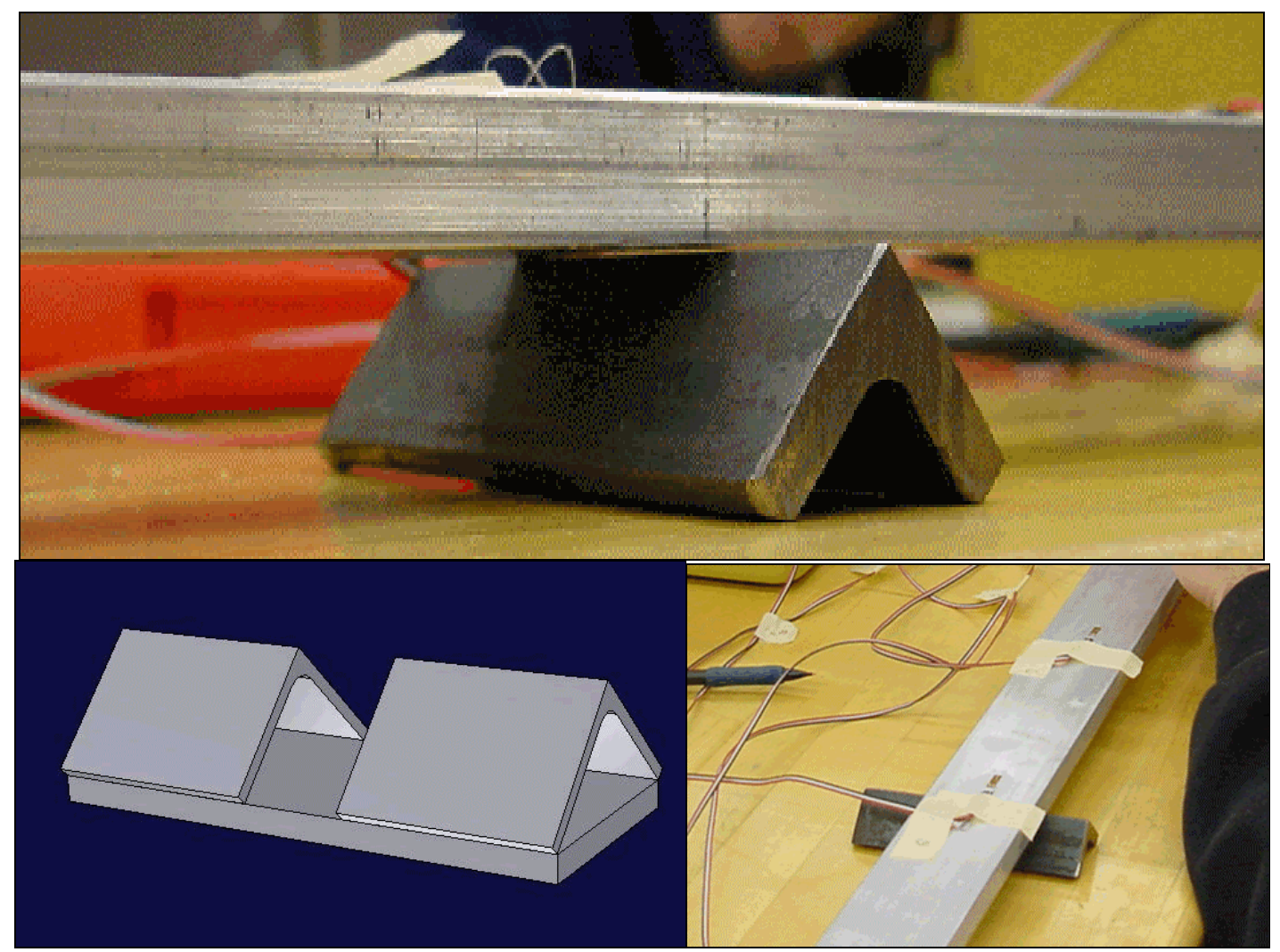

Figure 12: Beam support designs with original design on top, student design concept on lower left and implemented design configuration on lower right.

Figures 13 below depicts the instrumented overhung beam being tested for strain at the boundary condition offset, which represents the simply supported beam. Strain measurements were then analyzed to find the estimated optimal offset distance using interpolation techniques. As a future refinement, a strain gage will be mounted at the analytical optimal offset distance for more direct correlation of theory with experiment.

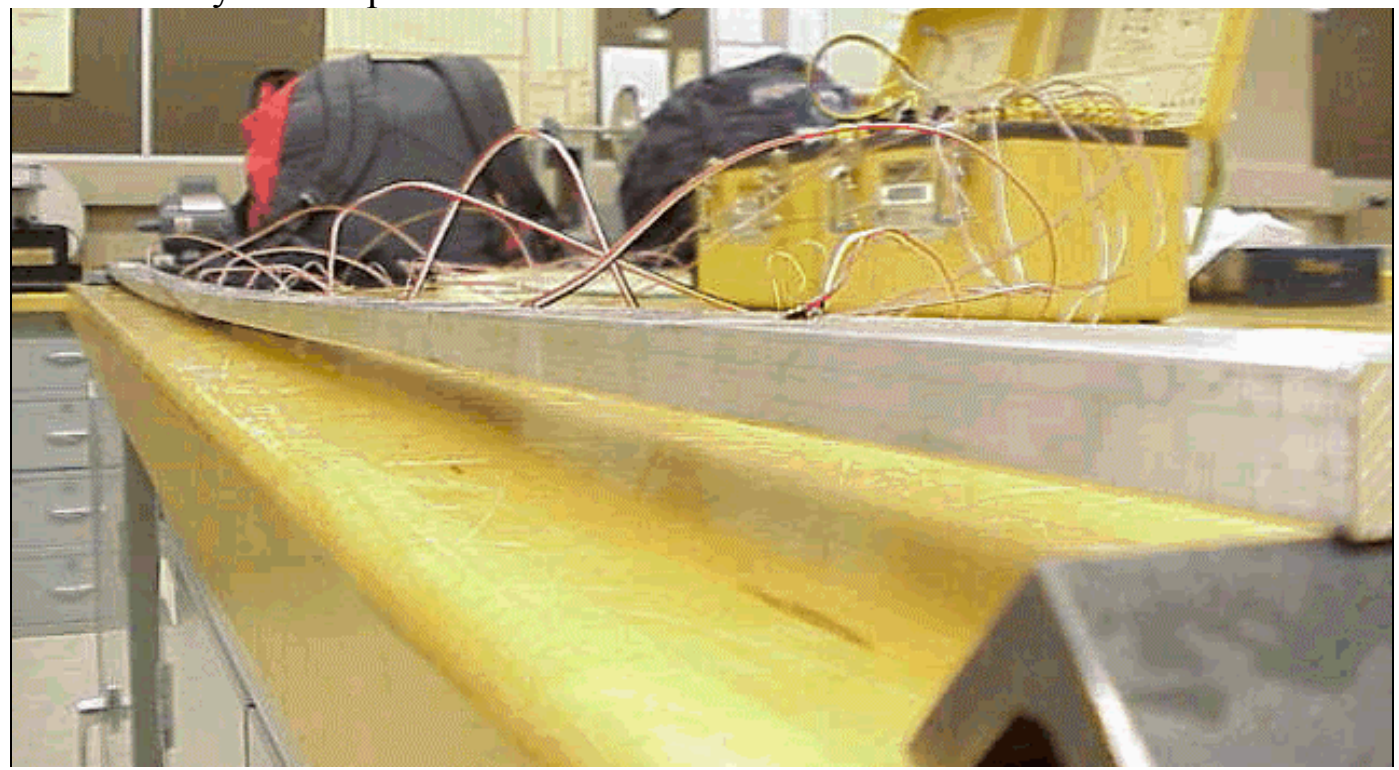

Figure 13: Overhung Beam supports and testing configuration with supports set to model a simply supported beam (e.g. offset distance, $a=0$ ") 


\section{Experimental Results for Deflection:}

The experimental results for deflection matched closely with both the theoretical and finite element analysis (FEA) results for offset distances, $\mathrm{a}=0,6,12,18,24,30,36,42$, and 48 inches respectively. The deflection results for the optimal offset distance of 19.882 inches (for the 96 inch long beam) is also shown. All of these results correlate closely with theory accounting for measurement error and provide the students with a hands-on experience that theory, experiment, and FEA can be closely correlated. Examples of deflection measurements with strain gages up and strain gages down are shown in Figure 14, an example of the finite element analysis results is shown in Figure 15, and a comparison table of Theory, Experiment, and Finite Element Analysis is shown in Figure 16. As seen in Figure 16, the deflection results indicate very good correlation between the Theory, Experiment, and FEA.
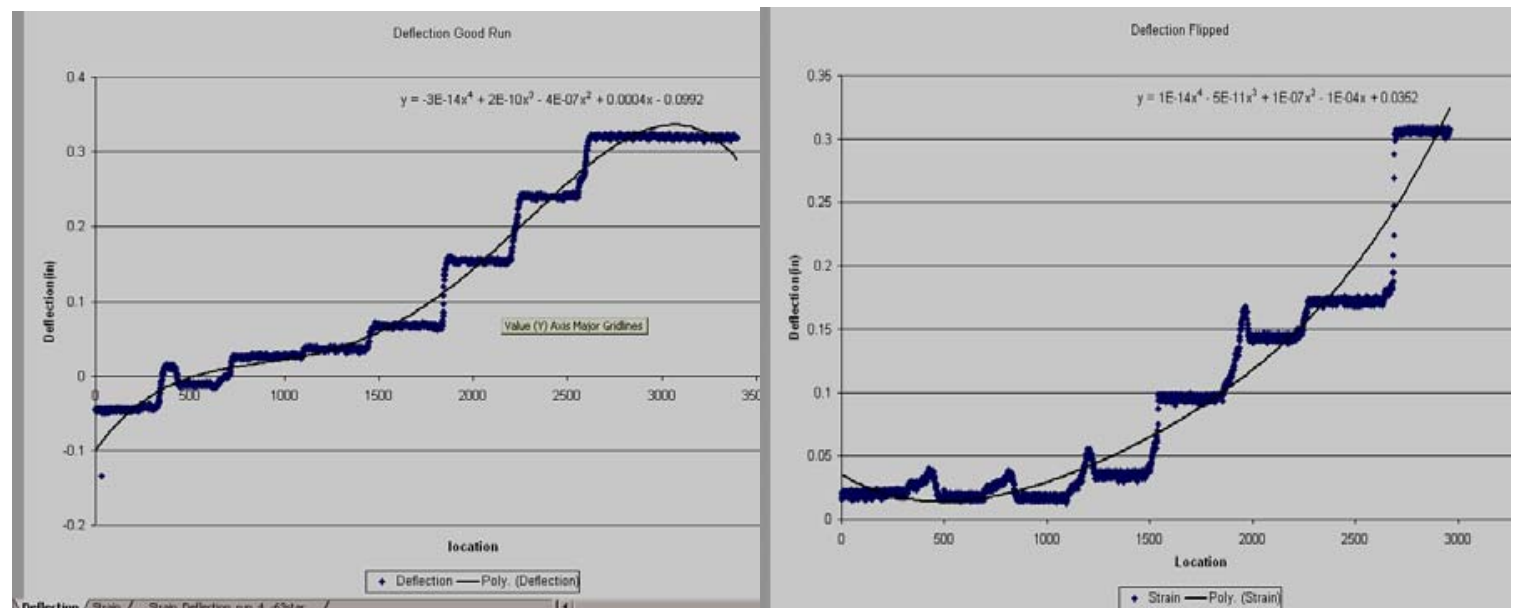

Figure 14 Example of deflection measurement for Simply Supported Beam with strain gages up (e.g. Compression on left) and strain gages down (e.g. Tension on right)

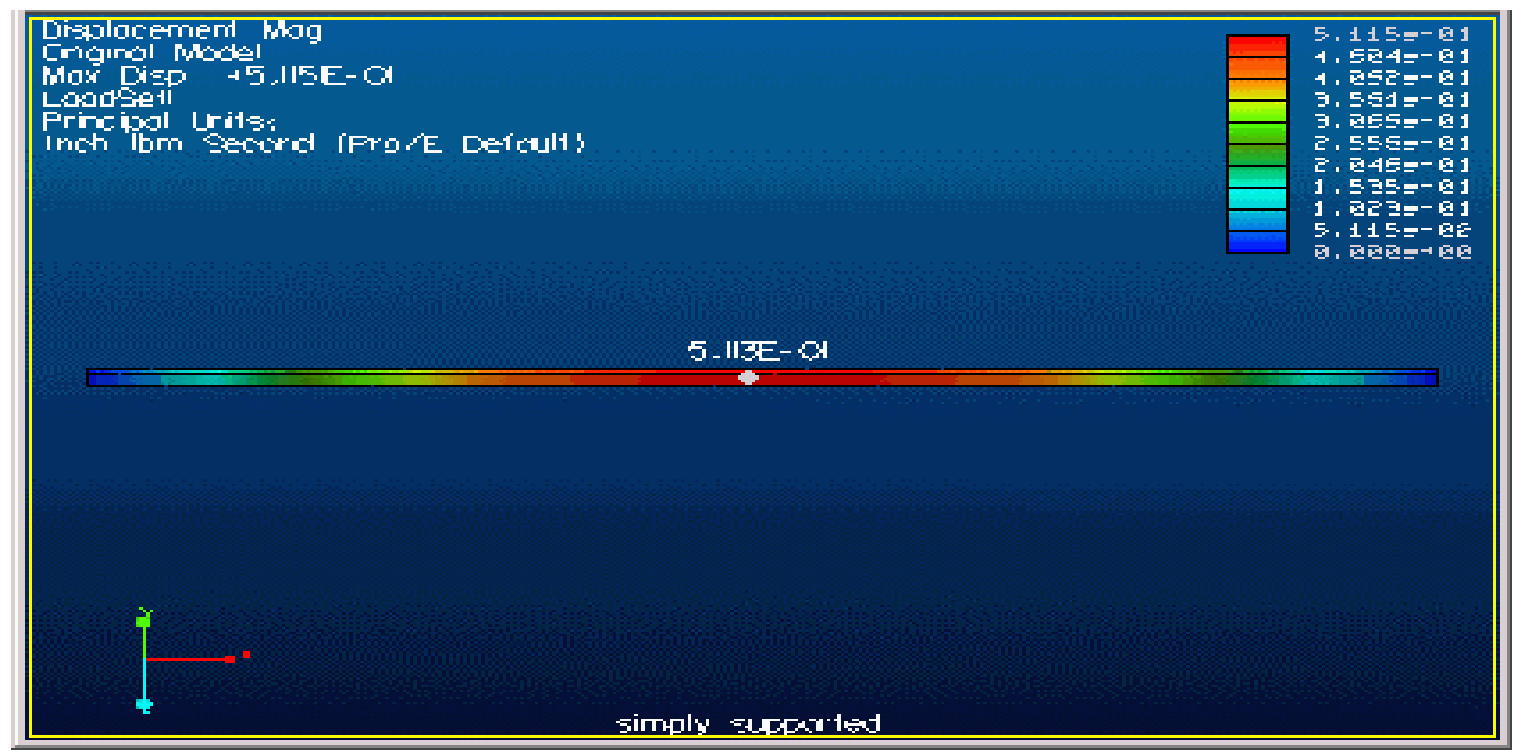

Figure 15 Example of finite element analysis deflection results for Simply Supported Beam 


\begin{tabular}{|l|c|c|c|}
\hline \multicolumn{1}{|c|}{ DEFLECTION RESULT COMPARISON } & Theoretical & Experimental & Finite Element Analysis \\
\hline 0 inch Support Offset Distance (e.g. & -.522 & -.518 & -.511 \\
Simply Supported Beam) (in) & -.299 & -.285 & -.295 \\
6 inch Support Offset Distance (in) & -.143 & -.150 & -.140 \\
12 inch Support Offset Distance (in) & -.045 & -.055 & -.044 \\
18 inch Support Offset Distance (in) & -.025 & -.024 & -.024 \\
19.882 inch Optimal Support Offset & -.007 & -.016 & -.006 \\
Distance (in) & .024 & .024 & .024 \\
24 inch Support Offset Distance (in) & .020 & .020 & .020 \\
30 inch Support Offset Distance (in) & .007 & .006 & .007 \\
36 inch Support Offset Distance (in) & .000 & .000 & .000 \\
42 inch Support Offset Distance (in) & & \\
48 inch Support Offset Distance (in) &
\end{tabular}

Figure 16: Midpoint deflection result comparison table.

\section{Experimental Results for Strain:}

The experimental results for strain very more varied than deflection, but still reasonably matched both the theoretical and finite element analysis (FEA) results for offset distances, a = 0, 6, 12, 18, $24,30,36,42$, and 48 inches respectively. The strain results for the optimal offset distance of 19.882 inches (for the 96 inch long beam) is also shown. The theoretical strain values are shown in Figure 17, a typical experimental result is shown in Figure 18, and a typical FEA result is shown in Figure 19, and a comparison table of Theory, Experiment, and Finite Element Analysis is shown in Figure 20. As seen in Figure 20, the deflection results indicate good correlation between the Theory, Experiment, and FEA.

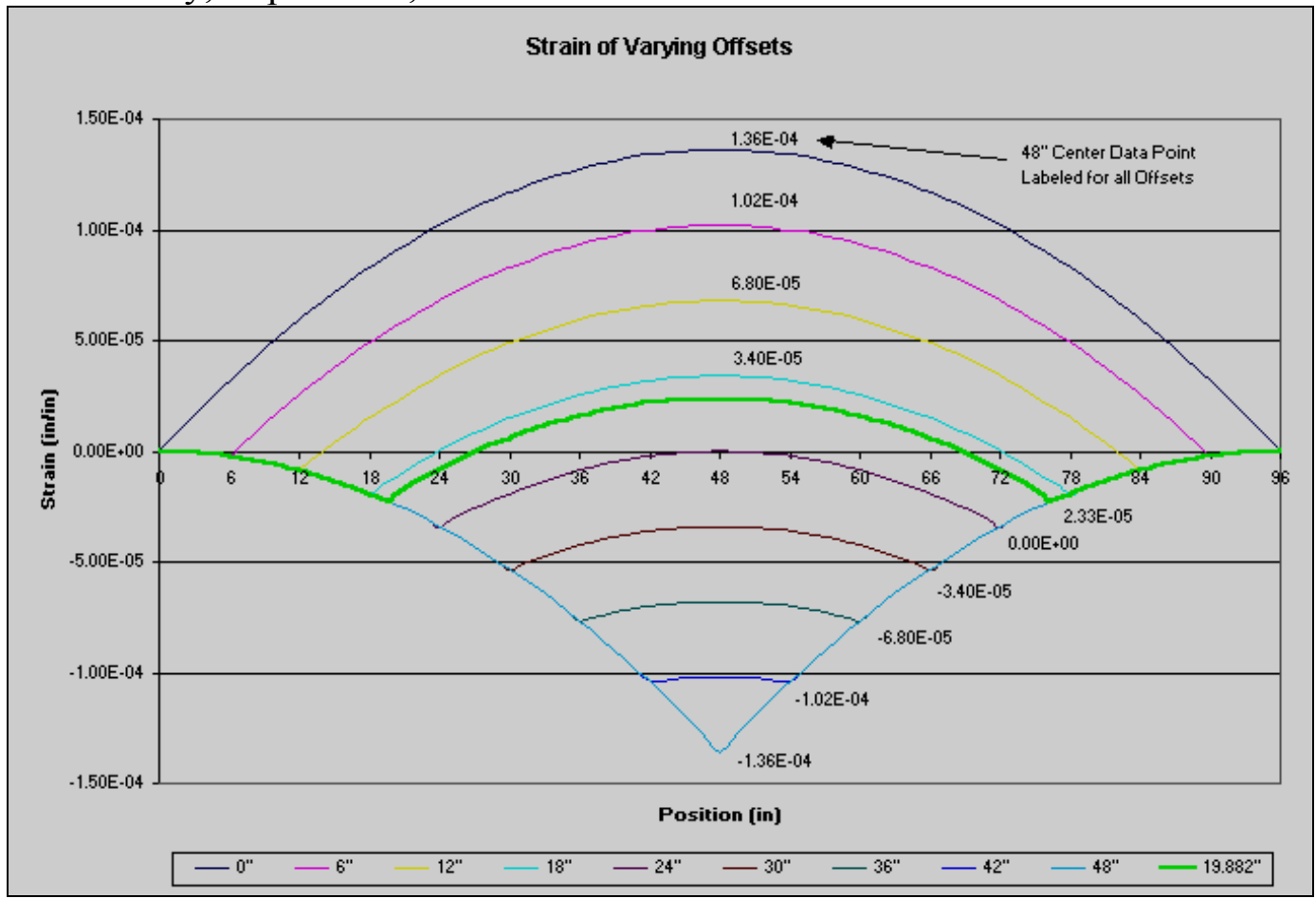

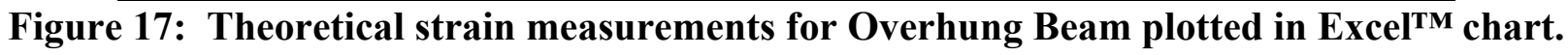




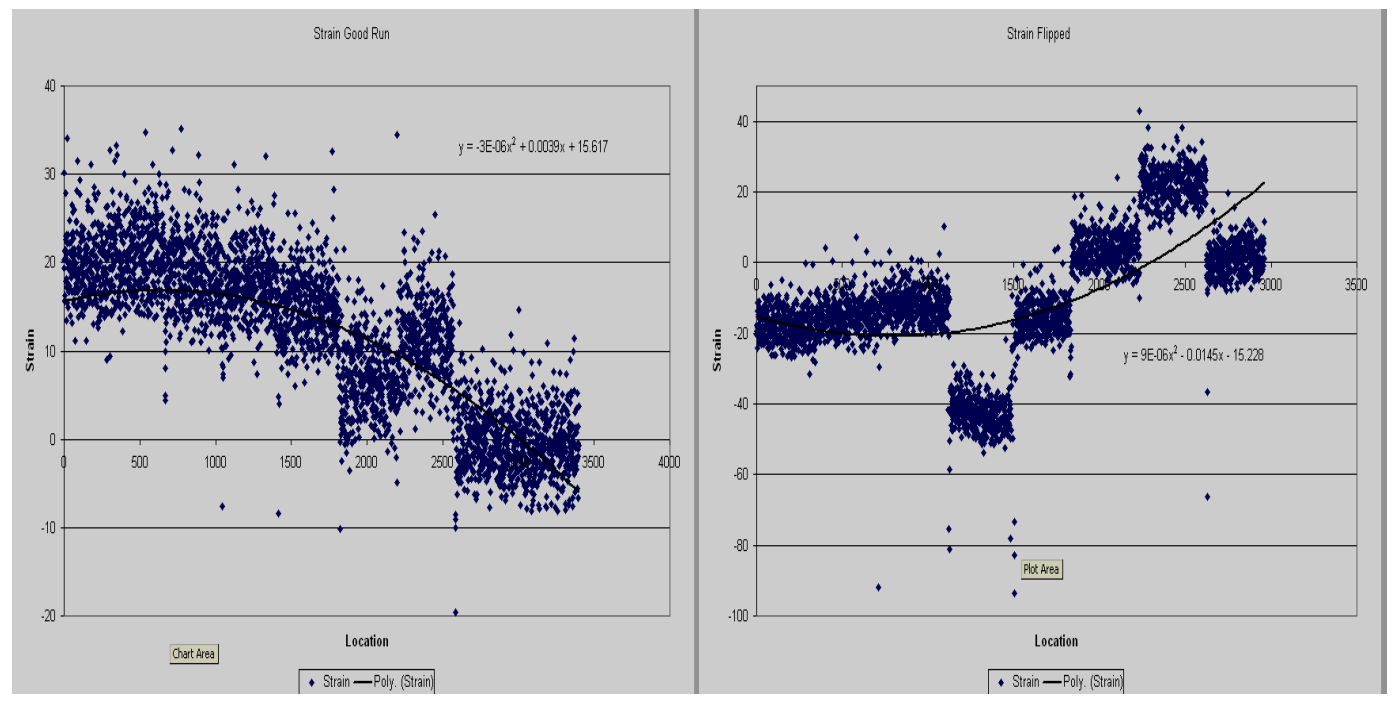

Figure 18 Experimental strain measurements for Simply Supported Beam with strain gages up (e.g. Compression on left) and strain gages down (e.g. Tension on right)

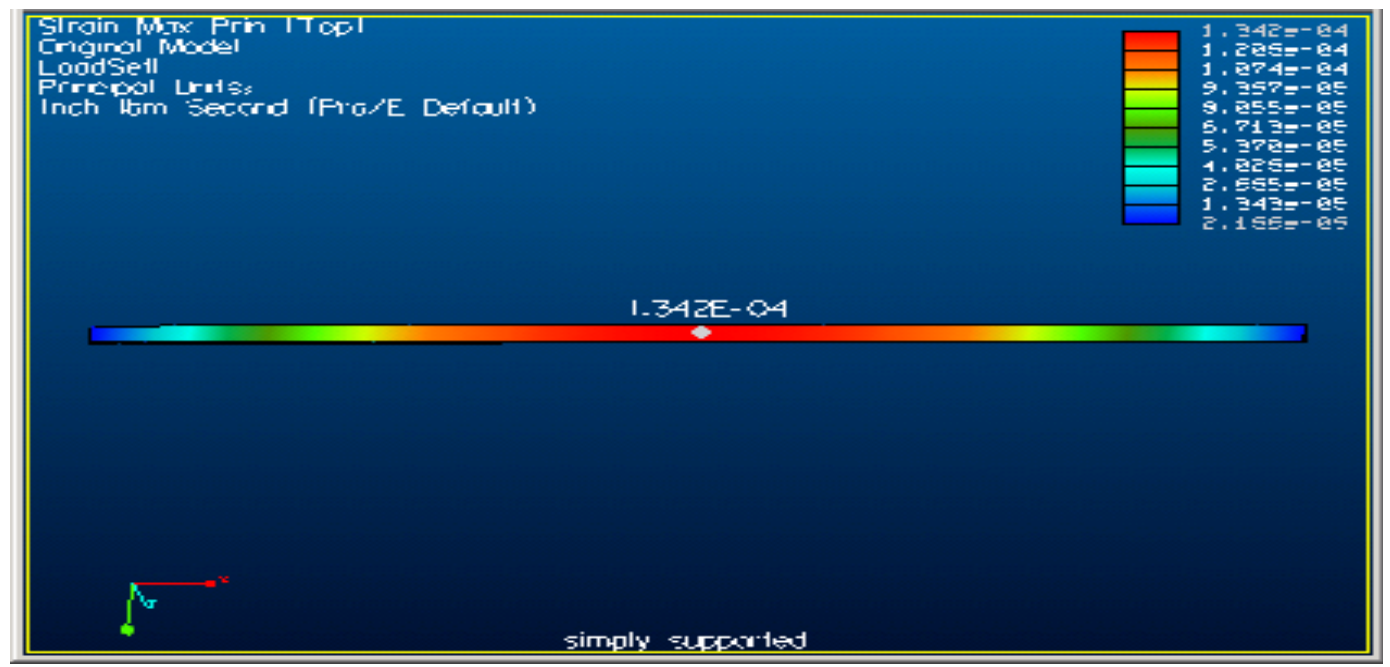

Figure 19: FEA strain results for Overhung Beam at zero offset.

\begin{tabular}{|l|c|c|c|}
\hline \multicolumn{1}{|c|}{ STRAIN RESULT COMPARISON } & Theoretical & Experimental & $\begin{array}{c}\text { Finite Element } \\
\text { Analysis }\end{array}$ \\
\hline 0 inch Support Offset Distance (e.g. & 136 & 188 & 134 \\
Simply Supported Beam) (ustrain) & 102 & 60 & 101 \\
6 inch Support Offset Distance (ustrain) & 68 & 31 & 67 \\
12 inch Support Offset Distance (ustrain) & 34 & 28 & 34 \\
18 inch Support Offset Distance (ustrain) & 23 & 27 & 23 \\
19.882 inch Optimal Support Offset & 0 & 3 & 0 \\
Distance (ustrain) & -34 & -11 & -34 \\
24 inch Support Offset Distance (ustrain) & -68 & -67 & -67 \\
30 inch Support Offset Distance (ustrain) & -102 & -115 & -101 \\
36 inch Support Offset Distance (ustrain) & -136 & -131 & -131 \\
42 inch Support Offset Distance (ustrain) & & \\
48 inch Support Offset Distance (ustrain) & -131 & \\
\hline
\end{tabular}

Figure 20: Midpoint strain result comparison table. 


\section{Conclusions:}

The overhung beam project has been shown to provide Experimental Mechanics students with a design, analysis, and test experience that brings mechanics theory to practice. Experimental results correlated to theory with improved results for deflection than for strain. Variations in strain gage mounting and instrumentation drift could have contributed to this result. The FEA results correlated extremely well to theory with less than a $5 \%$ difference.

This project also gave the students an open-ended design experience as they designed a support structure for the beam that would not crush the strain gage mounted at the center of the beam. Students modeled, fabricated, and put their design into action during the course of this experiment.

Future work will entail developing a support structure that mounts the beam at the neutral plane in an attempt to minimize the experimental error with respect to theoretical expectations. Future work will also involve utilizing optimal beam methodologies to contour the beam to minimize the weight of the beam using Solver ${ }^{\mathrm{TM}}$ and FEA software while providing surface constant stress.

\section{Bibliography:}

1. Shigley, J.E, Mischke, C.R., (2001), Mechanical Engineering Design $\left(6^{\text {th }}\right.$ ed.), N.Y., New York: McGraw-Hill.

2. Szaroletta, W.K, Introducing Single-Criterion Optimization Methods into Mechanics Classes, Proceedings of ASEE International Conference and Exposition 2002, Montreal, Quebec

3. Microsoft, Inc website (January, 2003), http://www.microsoft.com, Redmond, Washington

4. COSMOS, Inc website (January, 2003), http://www.cosmosm.com/dstar.htm. Frederick, Maryland.

5. IronCAD, Inc. website (January, 2003), http://www.ironcad.com, Los Angeles, California

\section{Biography:}

WILLIAM K. SZAROLETTA, P.E.

Professor Szaroletta is an assistant professor of mechanical engineering technology at Purdue University. A member of ASEE, he has 18 years industry experience in engineering and project management positions, with 12 awarded patents. He received his B.S. Degree in Mechanical Engineering from University of Michigan, Ann Arbor in 1977, M.S. Degree in Engineering (Product Design) from Stanford University in 1984, and a Master of Applied Mathematical Sciences Degree (Computer Science) from University of Georgia in 2000. He has 7 years university teaching experience, where his current applied research interests are rapid product design engineering, experimental mechanics laboratory automation, and applied optimization. 